\title{
DETECTION OF BIOFILM FORMATION AND ANTIBIOTIC RESISTANCE OF SALMONELLA IN BROILER CHICKEN
}

\author{
HEND K. SOROUR and FATMA AMER \\ Reference Laboratory for Veterinary Quality Control on Poultry Production, \\ Animal Health Research Institute
}

Received: 31 March 2018; Accepted: 30 April 2018

\begin{abstract}
Two hundred fifty samples from different broiler chicken flocks were subjected for isolation of salmonella species (2017-2018). The percentage for isolated Salmonella was 14.4\% (36/250). The obtained Salmonlla strains were obtained (Salmonella bardo, Salmonella norwich, Salmonella brancaster, Salmonella sekondi $\Pi$, Salmonella lamberhurst, Salmonella belgdam, Salmonella kentucky, Salmonella enteritidis, Salmonella goetebory, Salmonella kisii, Salmonella nigeria, Salmonella grampian, Salmonella newport, Salmonella noyo, Salmonella colindale, Salmonella seremban, Salmonella remo, Salmonella lindenburg, Salmonella a natum, Salmonella virchow, Salmonella tamiland, Salmonella york. In vitro antimicrobial sensitivity testing carried out on isolated salmonella strains revealed different antimicrobial resistance variation, high resistance rate were observed with lomefloxacin $(77.7 \%)$, tetracycline $(61 \%)$, kanamcin $(50 \%)$ and trimethoprime and levofloxacin $(47.2 \%)$. Also strains were subjected for detection of biofilm formation using glass tube test and detection of fim A gene was used for biofilm confirmation, 61.11\% (22/36) of strains was having ability to produce biofilm, while $38.88 \%$ (14/36) have no ability for biofilm production. Both positive and the negative biofilm formation of salmonella strains revealed the same degree of antibiotic resistance (100\%). No great significance between biofilm formation, multidrug resistance and the intensity of clinical signs and postmortem lesions were observed, so no relation between biofilm formation and antimicrobial resistance.
\end{abstract}

Key words: Biofilm, salmonella, antibiotic resistance, broiler.

\section{INTRODUCTION}

Salmonella can cause disease in domestic animals, differ in severity of a signs, diarrhea and enteritis to systemic syndrome, lead to great economic losses in poultry industry. Salmonellosis is of public health concern in both the developed and developing countries, it is one of the most important pathogens transmitted by food, especially poultry, which cause food poisoning, it has the ability to form biofilms on surfaces and It's adhesion can be influenced by different physicochemical properties of these surfaces, while Salmonella uses fimbriae and produces cellulose as the main matrix components of biofilms.

Salmonella infections are a serious medical and veterinary problem worldwide and there is an increasing need for new strategies for prevention and control (Majowicz et al., 2010).

Corresponding author: Dr. HEND K. SOROUR

E-mail address: drhendsorour@gmail.com

Present address: Reference Laboratory for Veterinary Quality Control on Poultry Production, Animal Health Research Institute
Biofilms are bacterial association that attach to a biological or non-biological surface and are enveloped by a bacterial-initiated matrix. This structure promote bacteria to survive in hostile conditions such as exposure to UV light, metal toxicity, acid exposure, dehydration and salinity, phagocytes, and several antibiotics and antimicrobial agents (Hall-Stoodley et al., 2004), as well as they can also form biofilms on chicken intestinal epithelium Ledeboer and Jones, (2005).

Microorganisms may be naturally resistant to antimicrobial agent or a specific category of antimicrobials but resistance may also be acquired. (Kadlec et al., 2012).

Many bacteria are able to attach and tocolonize environmental surfaces by producing biofilms (Donlan, and Costerton, 2002). Surface-associated community forming microcolonies surrounded by a matrix of exopolymers that trap other bacteria, nutrients, and debris is known as bacterial biofilm (Chavant et al., 2002).

The biofilm formation is required several developmental steps that included several 
distinguishable steps: (a) attachment to the carrier surface, reversible, (b) irreversible attachment, binding to the surface with the participation of adhesions or exopolysaccharides, (c) the development of microcolonies, a distinct mushroom shape, (d) the maturation of biofilmarchitecture (Donlan, and Costerton, 2002) and (Barnhart and Chapman, 2006), (e) under favorable conditions, the synthesis of martrix compoundsdecline and biofilm dispersion due to enzymatic cleavage of the matrix Gjermansen et al. (2005).

The fim A gene encodes the major structural subunit, while the fim $\mathrm{H}$ gene encodes the adhesin protein that is located at the tip of the assembled fimbrial structure and mediates binding to the receptor. The fim $\mathrm{H}$ adhesin is involved in biofilm formation on
HEp-2 tissue culture cells, murine intestinal epithelium, and chicken intestinal epithelium (Boddicker et al., 2002).

The aim of the work was to detect relation between antibiotic resistance of Salmonella strains and biofilm formation in broiler chicken.

\section{MATERIALS AND METHOD}

\section{1- Sampling}

Two hundred fifty samples obtained from different broiler flocks at different age and from different sources (farms, back yard, shops) were subjected for isolation of salmonella from (2017 to2018) as shown in table (1).

Table 1: Sampling for salmonella isolation from broiler flocks.

\begin{tabular}{ccccc}
\hline $\begin{array}{c}\text { Age of chicken } \\
\text { sample }\end{array}$ & $\begin{array}{c}\text { Number } \\
\text { of samples }\end{array}$ & organ & Clinical signs & $\begin{array}{c}\text { Postmortem } \\
\text { examination }\end{array}$ \\
\hline 1 day old & 50 & YolkLiver, heart & $\begin{array}{c}\text { Apparently } \\
\text { healthy }\end{array}$ & $\begin{array}{c}\text { Omphilites /perhepatitis } \\
\text { percarditis }\end{array}$ \\
\hline One week & 25 & Internal organs & Diarrhea & Greenish / Percarditis \\
\hline Two weeks & 25 & Ceacum & Diarrhea & Typhilitis \\
\hline Three weeks & 25 & Ceacum & Diarrhea & Typhilitis \\
\hline Four weeks & 25 & Internal organs & Diarrhea & Pale liver / Percarditis \\
\cline { 2 - 4 } & 50 & Cloacal swabs & Diarrhea & Diarrhea \\
\hline Total & 50 & Brain & $\begin{array}{c}\text { Nervous } \\
\text { Signs }\end{array}$ & $\begin{array}{c}\text { Diarrhea / inflammation } \\
\text { of brain }\end{array}$ \\
\hline
\end{tabular}

\section{2-Isolation}

Salmonella isolation and identification was done according to standard methods (ISO 6579:2002) and salmonella serotyping was done according to (Popoff, 2001).
3-Antimicrobial sensitivity test was carried out according to the Clinical and Laboratory Standards Institute (CLSI/NCCLS, 2009). Using disk diffusion method, Table (2).

Table2: Inhibition Zone Diameter Interpretive Standards Breakpoint for Enterobacteriaceae.

\begin{tabular}{|c|c|c|c|c|c|}
\hline \multirow{2}{*}{ Test group } & \multirow{2}{*}{$\begin{array}{c}\text { Antimicrobial } \\
\text { agent }\end{array}$} & \multirow{2}{*}{$\begin{array}{c}\text { Disk } \\
\text { content }\end{array}$} & \multicolumn{3}{|c|}{ Zone diameter nearest whole $\mathbf{m m}$} \\
\hline & & & Resistant(R) & Intermediate(I) & Sensitive(S) \\
\hline CEPHEMS & $\begin{array}{c}\text { Ceftriaxone } \\
\text { (CRO30) }\end{array}$ & $30 \mu \mathrm{g}$ & $\leq 13$ & 14-20 & $\geq 21$ \\
\hline \multirow[t]{4}{*}{ Aminoglycosides } & Gentamycin (CN 10) & $10 \mu \mathrm{g}$ & $\leq 12$ & 13-14 & $\geq 15$ \\
\hline & Amikacin (AK30) & 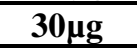 & $\leq \mathbf{1 4}$ & 15-16 & $\geq 17$ \\
\hline & Kanamycin (K30) & $30 \mu \mathrm{g}$ & $\leq \mathbf{1 3}$ & 13-14 & $\geq 15$ \\
\hline & Tobramycin (TOB10) & $10 \mu \mathrm{g}$ & $\leq 12$ & $12-14$ & $\geq 15$ \\
\hline \multirow{2}{*}{ Tetracyclines } & Tetracycline (TE30) & $30 \mu \mathrm{g}$ & $\leq \mathbf{1 1}$ & $12-14$ & $\geq 15$ \\
\hline & Doxycycline (DO30) & $30 \mu \mathrm{g}$ & $\leq \mathbf{1 0}$ & 11-13 & $\geq 14$ \\
\hline \multirow[t]{5}{*}{ Fluoroquinoiones } & Ciprofloxacin (CIP5) & $5 \mu \mathrm{g}$ & $\leq \mathbf{1 5}$ & 16-20 & $\geq 21$ \\
\hline & Levofloxacin (LEV 5) & $5 \mu \mathrm{g}$ & $\leq 13$ & 14-16 & $\geq 17$ \\
\hline & $\begin{array}{c}\begin{array}{c}\text { Lomefloxacin } \\
\text { (LOM10) }\end{array} \\
\end{array}$ & $10 \mu \mathrm{g}$ & $\leq 18$ & 19-21 & $\geq 22$ \\
\hline & Ofloxacin \OFX5 & $5 \mu \mathrm{g}$ & $\leq 12$ & 13-15 & $\geq 16$ \\
\hline & Norfloxacin \NOR10 & $5 \mu g$ & $\leq 12$ & 13-16 & $\geq 17$ \\
\hline $\begin{array}{l}\text { FOLATE Pathway } \\
\text { inhibitors }\end{array}$ & Trimethoprime $\backslash$ TR5 & $5 \mu \mathrm{g}$ & $\leq \mathbf{1 0}$ & 11-15 & $\geq 16$ \\
\hline
\end{tabular}




\section{4- Detection of salmonella biofilm formation}

A- Phenotypic test (glass test tube) according to (Daxin Peng, 2016).

The overnight cultures of each bacterium were diluted 1:100 in the diluted TSB. Two milliliters of each bacterial suspension were added into borosilicate glass test tubes and incubated at $28^{\circ} \mathrm{C}$ for $48 \mathrm{~h}$. Then the liquid was decanted and the tubes were washed gently three times with distilled water. Two $\mathrm{ml}$ of $0.4 \%$ crystal violet $(\mathrm{v} / \mathrm{v})$ were added into each tube and stained at room temperature for $20 \mathrm{~min}$.

\section{B- Conventional PCR technique. Extraction:}

DNA was extracted using commercially available kit, QIAamp® DNA Mini Kit, Catalogue no.51304

\section{PCR Reaction:}

The different primers used in this study are described in Table (3).

\section{PCR amplification.}

It was done in a $25 \mu \mathrm{l}$ reaction containing $12.5 \mu \mathrm{l}$ of Emerald Amp GT PCR master mix (2x premix), $1 \mu \mathrm{l}$ of each primer ( 20 pmol conc.), $4.5 \mu \mathrm{l}$ of PCR grade water, and $6 \mu \mathrm{l}$ of template. The cPCR reactions were performed in a Biometra T3 thermal cycler. The thermal profiles for fim $\mathrm{H}$ gene was applied according to (Hojati et al., 2015).

The PCR products were separated by electrophoresis on $1.5 \%$ agarose gel stained with ethidium bromide and photographed by a gel documentation system (Alpha Innotech, Biometra) ${ }^{\circledR}$.

Table 3: Oligonucleotide primers and sequences encoding for detection of biofilm formation using Fim H gene.

\begin{tabular}{|c|c|c|c|}
\hline Target gene & $\begin{array}{c}\text { Primers sequences } \\
5^{`}-3^{\prime}\end{array}$ & $\begin{array}{c}\text { Amplified } \\
\text { segment (bp) }\end{array}$ & Reference \\
\hline \multirow{2}{*}{ Fim $H$} & GTGCCAАTTCCTCTTACCGTT & \multirow{2}{*}{164} & \multirow[b]{2}{*}{ Hojati et al., 2015} \\
\hline & TGGAATAATCGTACCGTTGCG & & \\
\hline
\end{tabular}

\section{RESULTS}

Salmonella was detected in apparently healthy one day old broiler chicks that showed (Omphilites, perhepatitis, percarditis), also in diseased broilers that showed diarrhea, nervous signs, unable to walk showed greenish and paleness liver, percarditis, perhepatitis, typhilitis, enlarged cecum, inflammation of brain and oophritis in postmortem examination. Salmoella was representing $14.4(36 / 250)$ in different broiler flocks at different age from different localities (2017-2018).

Different salmonella strains was isolated, (Salmonella bardo, Salmonella norwich, Salmonella brancaster, Salmonella sekondiI, Salmonella lamberhurst, Salmonella belgdam) were demonstrated in one day old, while (Salmonella kentucky, Salmonella enteritidis, Salmonella goetebory, Salmonella kisii) demonstrated at one week age, also (Salmonella nigeria, Salmonella grampian) were reported at two weeks age, (Salmonella newport, Salmonella enteritidis, Salmonella noyo, Salmonella colindale) were at three weeks age, at fourth weeks (Salmonella seremban, Salmonella remo, Salmonella lindenburg, Salmonella kentucky, Salmonella enteritidis, Salmonella anatum, Salmonella virchow, Salmonella tamiland and Salmonella york as shown in Table (4).

Biofilm formation of salmonella strains was detected using a glass tube test, where Salmonella strains were tested for biofilm formation on glass surface. The positive biofilm formation were produced rings at the liquid-air interface on the glass test tube walls or produced color staining at the bottom of the tube and the confirmation was done using fim $\mathrm{H}$ gene (Hojati et al., 2015).

The percentage of $61.11 \%(22 / 36)$ of salmonella strains have the ability for biofilm producation, while 38.88 (14/36) have no ability for biofilm production Table (5), the positive biofilm formation was observed in Salmonella Kentucky (6/11) 54\%, Salmonella enteritidis (2/4) 50\% Seremban, Salmonella norwich, Salmonella lindenburg, Salmonella virchow (1/2) 50\%, Salmonella brancaster, Salmonella grampian, Salmonella belgam, Salmonella bardo, Salmonella york while no biofilm formation in Salmonella Kentucky (5/11) $45 \%$, Salmonella enteritidis (2/4)50\%, Salmonella goetebory, Salmonella anatum, Salmonella sekondi П, Salmonella lamberhurst, Salmonella virchow (1/2) $50 \%$, Salmonella noyo, Salmonella timiland Table (6).

The disk diffusion test revealed at the highest degree of resistance were observed with lomefloxacin $(77.7 \%)$, tetracycline $(61 \%)$, kanamcin $(50 \%)$ and trimethoprime and levofloxacin $(47.2 \%)$ and lowest resistance degree was observed with Ceftriaxone Table (7).

Antibiotic resistance was reported in both positive and negative biofilm formation in salmonella strains 
(100\%) and multidrug resistance was observed in the positive biofilm formation and negative biofilm formation.
Salmonella strains which had ability for biofilm was resistance to more than one antibiotics $17 / 22(72 \%)$ and Salmonella strains which have not ability for biofilm were resistance to more than one antibiotics 10/14 (71\%) Table (8),(9).

Table 4: prevalence of salmonella in broiler chickens.

\begin{tabular}{|c|c|c|c|c|c|c|c|}
\hline $\begin{array}{c}\text { Age of } \\
\text { chicken } \\
\text { samples }\end{array}$ & Number & $\begin{array}{c}\text { Site of } \\
\text { isolation }\end{array}$ & Signs/PM & $\begin{array}{c}\text { Number } \\
\text { of Positive }\end{array}$ & $\begin{array}{l}\text { Type of isolated } \\
\text { salmonella strain }\end{array}$ & $\begin{array}{c}\text { Number of } \\
\text { salmonella } \\
\text { strains }\end{array}$ & $\begin{array}{c}\text { Percentage } \\
\text { of } \\
\text { salmonella }\end{array}$ \\
\hline \multirow{6}{*}{$\begin{array}{l}1 \text { day } \\
\text { old }\end{array}$} & \multirow{6}{*}{50} & \multirow{6}{*}{$\begin{array}{l}\text { Yolk } \\
\text { Liver }\end{array}$} & \multirow{3}{*}{$\begin{array}{l}\text { Apparently } \\
\text { healthy }\end{array}$} & \multirow{6}{*}{650} & Salmonella bardo & $1 / 6$ & \multirow{6}{*}{12} \\
\hline & & & & & Salmonella norwich & $1 / 6$ & \\
\hline & & & & & Salmonella brancaster & $1 / 6$ & \\
\hline & & & \multirow{3}{*}{$\begin{array}{c}\text { Omphilites } \\
\text { /perhepatitis } \\
\text { percarditis }\end{array}$} & & Salmonella secondi $\Pi$ & $1 / 6$ & \\
\hline & & & & & Salmonella lamberhurst & $1 / 6$ & \\
\hline & & & & & Salmonella belgdam & $1 / 6$ & \\
\hline \multirow{4}{*}{$\begin{array}{l}\text { One } \\
\text { week }\end{array}$} & \multirow{4}{*}{25} & \multirow{4}{*}{$\begin{array}{l}\text { Internal } \\
\text { organs }\end{array}$} & \multirow{2}{*}{ Diarrhea } & \multirow{4}{*}{$5 / 25$} & Salmonella kentucky & $2 / 5$ & \multirow{4}{*}{20} \\
\hline & & & & & Salmonella enteritidis & $1 / 5$ & \\
\hline & & & Greenish liver / & & Salmonella goetebory & $1 / 5$ & \\
\hline & & & Percarditis & & Salmonella kisii & $1 / 5$ & \\
\hline \multirow{2}{*}{$\begin{array}{c}\text { Two } \\
\text { weeks }\end{array}$} & \multirow[b]{2}{*}{25} & \multirow[b]{2}{*}{ Ceacum } & Diarrhea & \multirow[b]{2}{*}{$2 / 25$} & Salmonella nigeria & $1 / 2$ & \multirow[b]{2}{*}{8} \\
\hline & & & $\begin{array}{c}\text { Typhilitis/ } \\
\text { enlarged cecum }\end{array}$ & & Salmonella grampian & $1 / 2$ & \\
\hline \multirow{4}{*}{$\begin{array}{l}\text { Three } \\
\text { weeks }\end{array}$} & \multirow{4}{*}{25} & \multirow{4}{*}{ Ceacum } & \multirow{2}{*}{ Diarrhea } & \multirow{4}{*}{$4 / 25$} & Salmonella newport & $1 / 4$ & \multirow{4}{*}{16} \\
\hline & & & & & Salmonella enteritidis & $1 / 4$ & \\
\hline & & & \multirow{2}{*}{$\begin{array}{c}\text { Typhilitis/ } \\
\text { enlarged cecum }\end{array}$} & & Salmonella noyo & $1 / 4$ & \\
\hline & & & & & Salmonella colindale & $1 / 4$ & \\
\hline \multirow{12}{*}{$\begin{array}{l}\text { Four } \\
\text { weeks }\end{array}$} & \multirow{5}{*}{25} & \multirow{5}{*}{$\begin{array}{l}\text { Internal } \\
\text { organs }\end{array}$} & \multirow{2}{*}{ Diarrhea } & \multirow{5}{*}{$5 / 25$} & Salmonella seremban & $1 / 5$ & \\
\hline & & & & & Salmonella remo & $1 / 5$ & \\
\hline & & & & & Salmonella lindenburg & $1 / 5$ & 20 \\
\hline & & & Greenish liver / & & Salmonella kentucky & $1 / 5$ & \\
\hline & & & & & Salmonella enteritidis & $1 / 5$ & \\
\hline & & & & & Salmonella kentucky & $5 / 10$ & \\
\hline & & Cloacal & & & Salmonella anatum & $\mathbf{1 / 1 0}$ & 0 \\
\hline & 50 & swabs & Diarrhea & $10 / 50$ & Salmonella enteritidis & $1 / 10$ & 9 \\
\hline & & & & & Salmonella virchow & $2 / 10$ & \\
\hline & & & Diongh & & Salmonella kentucky & $3 / 5$ & \\
\hline & 50 & Brain & Narvanc Sirne & $5 / 50$ & Salmonella tamilandu & $1 / 5$ & 20 \\
\hline & & Bram & $\begin{array}{l}\text { Nervous Signs } \\
\text { Unable to walk }\end{array}$ & & Salmonella york & $1 / 5$ & \\
\hline Total & 250 & & & $36 / 250$ & & & 14.4 \\
\hline
\end{tabular}

Table 5: Detection of biofilm formation by salmonella strains.

\begin{tabular}{lcccc}
\hline \multicolumn{1}{c}{ Test } & $\begin{array}{c}\text { Number of } \\
\text { positive biofilm } \\
\text { formation }\end{array}$ & $\%$ & $\begin{array}{c}\text { Number of negative } \\
\text { biofilm formation }\end{array}$ & $\%$ \\
\hline $\begin{array}{l}\text { A.Phenotypic charaterization } \\
\text { Tube agglutination test }\end{array}$ & $22 / 36$ & $61.11 \%$ & $14 / 36$ & 38.88 \\
\hline B.Polymerase chain reaction test & $22 / 36$ & $61.11 \%$ & $14 / 36$ & 38.88 \\
\hline 1-fim $\mathrm{H}$ gene & & & \\
\hline
\end{tabular}

Number of salmonella (36) 
Table 6: Percentage of isolated salmonella strain.

\begin{tabular}{|c|c|c|c|c|c|c|}
\hline \multirow[b]{2}{*}{$\begin{array}{c}\text { Salmonella } \\
\text { Serotype }\end{array}$} & \multirow[b]{2}{*}{ Antigenic structure } & \multirow[b]{2}{*}{$\begin{array}{c}\text { Total } \\
\text { number }\end{array}$} & \multicolumn{4}{|c|}{ Numbers } \\
\hline & & & $\begin{array}{c}\text { Positive } \\
\text { salmonella } \\
\text { biofilm } \\
\text { formation }\end{array}$ & $\%$ & $\begin{array}{c}\text { Negative } \\
\text { salmonella } \\
\text { biofilm } \\
\text { formation }\end{array}$ & $\%$ \\
\hline Salmonella kentucky & O8,20,I,Z60 & 11 & 6 & $54 \%$ & 5 & 45.4 \\
\hline Salmonella seremban & O9,12,I,1,5 & 1 & 1 & 100 & $\mathbf{0}$ & $\mathbf{0}$ \\
\hline Salmonella norwich & O6,8,e,h,1,6 & 1 & 1 & 100 & $\mathbf{0}$ & $\mathbf{0}$ \\
\hline Salmonella lindenburg & O6,8,I,1,2 & 1 & 1 & 100 & $\mathbf{0}$ & $\mathbf{0}$ \\
\hline Salmonella virchow & O6,7,14,r,1,2 & 2 & 1 & 50 & 1 & 50 \\
\hline Salmonella brancaster & O3, O10,e,n,x,1,7 & 1 & 1 & 100 & $\mathbf{0}$ & $\mathbf{0}$ \\
\hline Salmonella grampian & O6,7,r,l,w & 1 & 1 & 100 & $\mathbf{0}$ & $\mathbf{0}$ \\
\hline Salmonella sekondi I & 01, 04, 012, 027, $\mathrm{z}_{29,-}$ & 1 & $\mathbf{0}$ & $\mathbf{0}$ & 1 & 100 \\
\hline Salmonella belgdam & O9,12,G,m,s,- & 1 & 1 & 100 & $\mathbf{0}$ & $\mathbf{0}$ \\
\hline Salmonella york & O9,12;Z28;enZ15 & 1 & 1 & 100 & $\mathbf{0}$ & $\mathbf{0}$ \\
\hline Salmonella bardo & O8,e,h,1,2 & 1 & 1 & 100 & $\mathbf{0}$ & $\mathbf{0}$ \\
\hline Salmonella enteritidis & O1,9,12,g,m;- & 4 & 2 & 50 & 2 & 50 \\
\hline Salmonella goetebory & O9,12,c,1,5 & 1 & $\mathbf{0}$ & $\mathbf{0}$ & 1 & 100 \\
\hline Salmonella anatum & O3,10;e,h,1,6 & 1 & $\mathbf{0}$ & $\mathbf{0}$ & 1 & 100 \\
\hline Salmonella lamberhurst & O3,10,e,h,e,n,z 15 & 1 & $\mathbf{0}$ & 100 & 1 & 100 \\
\hline Salmonella nigeria & O6,7,r,1,6 & 1 & 1 & 100 & $\mathbf{0}$ & $\mathbf{0}$ \\
\hline Salmonella colindale & $06,7, r, 1,7$ & 1 & 1 & 100 & $\mathbf{0}$ & $\mathbf{0}$ \\
\hline Salmonella noyo & O8,r,1,7 & 1 & $\mathbf{0}$ & $\mathbf{0}$ & 1 & 100 \\
\hline Salmonella kisii & O6,7;d;1,2 & 1 & 1 & 100 & $\mathbf{0}$ & $\mathbf{0}$ \\
\hline Salmonella newport & O6,8,20,e,h,1,2 & 1 & 1 & 100 & $\mathbf{0}$ & $\mathbf{0}$ \\
\hline Salmonella remo & O 1.4,12,27,r,1,7 & 1 & 1 & 100 & $\mathbf{0}$ & $\mathbf{0}$ \\
\hline Salmonella tamilandu & O 6,7, $z_{41}, z_{35}$ & 1 & $\mathbf{0}$ & $\mathbf{0}$ & 1 & $\mathbf{0}$ \\
\hline
\end{tabular}

Table 7: Antibiotic resistance profile for examined salmonella.

\begin{tabular}{|c|c|c|}
\hline Antibiotic disk & $\begin{array}{l}\text { Number of resistance antibiotic } \\
\text { to isolated salmonella }\end{array}$ & $\%$ \\
\hline \multicolumn{2}{|l|}{ CEPHEMS } & \multirow{2}{*}{22.2} \\
\hline Ceftriaxone $\backslash \mathrm{CRO}_{30}$ & $8 / 36$ & \\
\hline \multicolumn{3}{|l|}{ Aminoglycosides } \\
\hline$\overline{1-G e n t a m y c i n ~} \backslash \mathrm{CN} 10$ & $11 / 36$ & 30.5 \\
\hline 2-Amikacin $\backslash \mathrm{AK}_{30}$ & $13 / 36$ & 36.1 \\
\hline 3-Kanamycin $\backslash \mathrm{K}_{30}$ & $18 / 36$ & 50 \\
\hline 4-Tobramycin $\backslash \mathrm{TOB}_{10}$ & $11 / 36$ & 30.5 \\
\hline \multicolumn{3}{|l|}{ Tetracyclines } \\
\hline 1-Tetracyclinel $\mathrm{TE}_{30}$ & $22 / 36$ & 61 \\
\hline 2-Doxycycline $\backslash \mathrm{DO}_{30}$ & $10 / 36$ & 27.7 \\
\hline \multicolumn{3}{|l|}{ Fluoroquinoiones } \\
\hline 1-Ciprofloxacin $\backslash \mathrm{CIP}_{5}$ & $15 / 36$ & 41.6 \\
\hline 2-Levofloxacin $\backslash \mathrm{LEV}_{5}$ & $17 / 36$ & 47.2 \\
\hline 3-Lomefloxacin $\backslash \mathrm{LOM}_{10}$ & $28 / 36$ & 77.7 \\
\hline 4-Ofloxacin $\backslash \mathrm{OFX}_{5}$ & $15 / 36$ & 41.6 \\
\hline 5-Norfloxacin $\backslash \mathrm{NOR}_{10}$ & $10 / 36$ & 27.7 \\
\hline \multicolumn{3}{|l|}{ FOLATE Pathway inhibitors } \\
\hline Trimethoprime $\backslash \mathrm{TR}_{5}$ & $17 / 36$ & 47.2 \\
\hline
\end{tabular}


Table 8: Detection relation between antibiotic resistant and positive salmonella biofilm formation.

\begin{tabular}{|c|c|c|c|}
\hline & Salmonella strains & Antibiotic resistance & $\mathrm{ABCs} \%$ \\
\hline 1 & Salmonella seremban * & $\begin{array}{l}\mathrm{CRO}_{30}, \mathrm{~K}_{30}, \mathrm{TE}_{30}, \mathrm{DO}_{30}, \mathrm{CIP}_{5}, \mathrm{LEV}_{5}, \mathrm{LOM}_{10}, \mathrm{OFX}_{5}, \\
\mathrm{NOR}_{10}, \mathrm{TR}_{5}\end{array}$ & 10/13(76.9) \\
\hline 2 & Salmonella kentucky * & $\begin{array}{l}\mathrm{K}_{30}, \mathrm{TE}_{30}, \mathrm{DO}_{30}, \mathrm{CIP}_{5}, \mathrm{LEV}_{5}, \mathrm{LOM}_{10}, \mathrm{OFX}_{5}, \mathrm{NOR}_{10} \\
\mathrm{TR}_{5}\end{array}$ & $9 / 13(69.2)$ \\
\hline 3 & Salmonella norwich $*$ & $\mathbf{A K}_{30}, \mathbf{K}_{30}, \mathbf{T E}_{30}, \mathbf{L E V}_{5}, \mathbf{L O M}_{10}$ & 5/13(38.4) \\
\hline 4 & Salmonella kentucky * & $\mathrm{K}_{30}, \mathrm{TE}_{30}, \mathrm{CIP}_{5}, \mathrm{LEV}_{5}, \mathrm{LOM}_{10}, \mathrm{OFX}_{5}, \mathrm{NOR}_{10}, \mathrm{TR}_{5}$ & $8 / 13(61.5)$ \\
\hline 5 & Salmonella lindenburg * & $\mathrm{K}_{30}, \mathrm{TE}_{30}, \mathrm{CIP}_{5}, \mathrm{LEV}_{5}, \mathrm{LOM}_{10}, \mathrm{OFX}_{5}, \mathrm{NOR}_{10}, \mathrm{TR}_{5}$ & $8 / 13(61.5)$ \\
\hline 6 & Salmonella virchow * & $\begin{array}{l}\mathrm{CN}_{10}, \mathrm{~K}_{30}, \mathrm{TE}_{30}, \mathrm{CIP}_{5}, \mathrm{LEV}_{5}, \mathrm{LOM}_{10}, \mathrm{OFX}_{5}, \mathrm{NOR}_{10} \\
\mathrm{TR}_{5}\end{array}$ & $9 / 13(69.2)$ \\
\hline 7 & Salmonella brancaster* & $\mathbf{A K}_{\mathbf{3 0}}, \mathbf{K}_{\mathbf{3 0}}, \mathbf{T O B}_{10}, \mathbf{T E}_{\mathbf{3 0}}, \mathbf{L O M}_{10}, \mathbf{T R}_{\mathbf{5}}$ & $6 / 13(46.1)$ \\
\hline 8 & Salmonella grampian * & $\mathbf{C N}_{10}, \mathbf{A K}_{30}, \mathbf{T O B}_{10}, \mathbf{T E}_{30}, \mathbf{L O M}_{10}, \mathbf{T R}_{5}$ & 6/13(46.1) \\
\hline 9 & Salmonella sekondi II ${ }^{\mathrm{N}}$ & $\mathrm{CRO}_{30}, \mathrm{CN}_{10}, \mathrm{AK}_{30}, \mathrm{DO}_{30}, \mathrm{LEV}_{5}, \mathrm{LOM}_{10}$ & 6/13(46.1) \\
\hline 10 & Salmonella kentucky* & $\mathrm{TOB}_{10}, \mathrm{DO}_{30}, \mathrm{CIP}_{5}, \mathrm{LOM}_{10}, \mathrm{OFX}_{5}$ & 5/13(38.4) \\
\hline 11 & Salmonella kentucky $^{\mathrm{N}}$ & $\mathrm{TOB}_{10}, \mathrm{DO}_{30}, \mathrm{CIP}_{5}, \mathrm{LOM}_{10}, \mathrm{OFX}_{5}$ & $5 / 13(38.4)$ \\
\hline 12 & Salmonella kentucky $^{\mathrm{N}}$ & $\mathrm{CRO}_{30}, \mathrm{AK}_{30}, \mathrm{~K}_{30}, \mathrm{LEV}_{5}, \mathrm{LOM}_{10}$ & $5 / 13(38.4)$ \\
\hline 13 & Salmonella belgdam * & $\mathrm{CN}_{10}, \mathrm{~K}_{30}, \mathrm{TE}_{30}, \mathrm{CIP}_{5}, \mathrm{LEV}_{5}, \mathrm{LOM}_{10}, \mathrm{OFX}_{5}, \mathrm{NOR}_{10}$ & $8 / 13(61.5)$ \\
\hline 14 & Salmonella york* & $\mathrm{CN}_{10}, \mathrm{AK}_{30}, \mathrm{~K}_{30}, \mathrm{TOB}_{10}, \mathrm{TE}_{30}, \mathrm{CIP}_{5}$ & 6/13(46.1) \\
\hline 15 & Salmonella kentucky* & $\mathrm{K}_{30}, \mathrm{TOB}_{10}, \mathrm{TE}_{30}, \mathrm{DO}_{30}, \mathrm{CIP}_{5}, \mathrm{LEV}_{5}, \mathrm{LOM}_{10}, \mathrm{OFX}_{5}$ & $8 / 13(61.5)$ \\
\hline 16 & Salmonella bardo * & $\begin{array}{l}\mathrm{CN}_{10}, \mathrm{AK}_{30}, \mathrm{~K}_{30}, \mathrm{TOB}_{10}, \mathrm{TE}_{30}, \mathrm{CIP}_{5}, \mathrm{LEV}_{5}, \mathrm{LOM}_{10}, \\
\mathrm{OFX}_{5}, \mathrm{NOR}_{10}\end{array}$ & 10/13(76.9) \\
\hline 17 & Salmonella kentucky* & $\mathrm{CRO}_{30}, \mathrm{AK}_{30}, \mathrm{LEV}_{5}, \mathrm{LOM}_{10}, \mathrm{TR}_{5}$ & $5 / 13(38.4)$ \\
\hline 18 & Salmonella enteritidis* & $\mathbf{C N}_{10}, \mathbf{T O B}_{10}, \mathbf{T E}_{30}, \mathbf{D O}_{30}, \mathbf{L O M}_{10}, \mathbf{T R}_{5}$ & $6 / 13(46.1)$ \\
\hline 19 & Salmonella enteritidis $^{\mathrm{N}}$ & $\mathrm{CN}_{10}, \mathbf{T O B}_{10}, \mathrm{TE}_{30}, \mathbf{D O}_{30}, \mathrm{LOM}_{10}, \mathbf{T R}_{5}$ & 6/13(46.1) \\
\hline 20 & Salmonella kentucky ${ }^{\mathrm{N}}$ & $\begin{array}{l}\text { CRO30, CN } \\
\mathrm{LOM}_{10}, \mathrm{AK}_{30}, \mathrm{~K}_{30}, \mathrm{TOB}_{\mathbf{5}}, \mathrm{NOR}_{10}, \mathrm{TE}_{30}, \mathrm{CIP}_{5}, \mathrm{LEV} \\
\mathbf{5}\end{array}$ & 11/13(84.6) \\
\hline 21 & Salmonella enteritidis ${ }^{\mathrm{N}}$ & $\mathrm{CRO}_{30}, \mathrm{LOM}_{10}$ & $2 / 13(15.3)$ \\
\hline 22 & Salmonella goetebory $^{\mathrm{N}}$ & $\mathrm{CRO}_{30}, \mathrm{TE}_{30}, \mathrm{LEV}_{5}, \mathrm{LOM}_{10}, \mathrm{OFX}_{5} \mathrm{NOR}_{10}, \mathrm{TR}_{5}$ & 7/13(53.8) \\
\hline 23 & Salmonella kentucky $^{\mathrm{N}}$ & $\mathrm{K}_{30}, \mathrm{TE}_{30}, \mathrm{DO}_{30}, \mathrm{CIP}_{5}, \mathrm{LEV}_{5}, \mathrm{LOM}_{10}, \mathrm{OFX}_{5}$ & 7/13(53.8) \\
\hline 24 & Salmonella kentucky $^{\mathrm{N}}$ & $\mathrm{CRO}_{30}, \mathrm{AK}_{30}, \mathrm{~K}_{30}, \mathrm{LEV}_{5}, \mathrm{LOM}_{10}$ & $5 / 13(38.4)$ \\
\hline 25 & 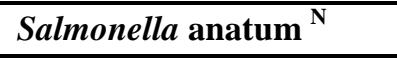 & $\mathrm{AK}_{30}, \mathrm{TE}_{30}, \mathrm{DO}_{30}, \mathrm{CIP}_{5}, \mathrm{LOM}_{10}$ & $5 / 13(38.4)$ \\
\hline 26 & Salmonella lamberhurst ${ }^{\mathrm{N}}$ & $\mathbf{A K _ { 3 0 }}, \mathbf{T E}_{\mathbf{3 0}}, \mathbf{T R}_{\mathbf{5}}$ & $3 / 13(23)$ \\
\hline 27 & Salmonella virchow ${ }^{\mathrm{N}}$ & $\mathrm{CN}_{10}, \mathrm{TE}_{30}, \mathrm{CIP}_{5}, \mathrm{LEV}_{5}, \mathrm{LOM}_{10}, \mathrm{OFX}_{5}, \mathrm{NOR}_{10}, \mathrm{TR}_{5}$ & 8/13(61.5) \\
\hline 28 & Salmonella kentucky* & $\mathrm{DO}_{30}, \mathrm{CIP}_{5}, \mathrm{LOM}_{10}, \mathrm{OFX}_{5}$ & $4 / 13(30)$ \\
\hline 29 & Salmonella remo * & $\mathrm{CN}_{10}, \mathrm{AK}_{30}, \mathrm{LOM}_{10}$ & $3 / 13(23 \%)$ \\
\hline 30 & Salmonella newport * & $\mathrm{CN}_{10}, \mathrm{~K}_{30}, \mathrm{TOB}_{10}, \mathrm{TE}_{30}$ & $4 / 13(30 \%)$ \\
\hline 31 & Salmonella nigeria * & $\mathbf{A K}_{30}, \mathbf{T O B}_{10}, \mathbf{T R}_{\mathbf{5}}$ & $3 / 13(23 \%)$ \\
\hline 32 & Salmonella enteritidis* & $\mathbf{A K}_{30}, \mathbf{T E}_{30}, \mathbf{T R}_{5}$ & $3 / 13(23 \%)$ \\
\hline 33 & Salmonella colindale * & $\mathbf{A} \mathbf{K}_{\mathbf{3 0}}, \mathbf{T R}_{\mathbf{5}}$ & $2 / 13(15.3 \%)$ \\
\hline 34 & Salmonella noyo ${ }^{\mathrm{N}}$ & $\mathbf{A K}_{\mathbf{3 0}}, \mathbf{T R}_{\mathbf{5}}$ & $2 / 13(15.3 \%)$ \\
\hline 35 & S.Tamilandu ${ }^{\mathrm{N}}$ & $\mathbf{L O M}_{10}$ & $1 / 13(7.69 \%)$ \\
\hline & Salmonella kisii* & $\mathrm{CN}_{10}, \mathrm{K30}, \mathrm{LOM}_{10}$ & $3 / 13(7.6 \%)$ \\
\hline
\end{tabular}

* Positive for biofilm (use glass tube and fim $\mathrm{H}$ gene)

N Negative for biofilm formation (glass tube test and fim $\mathrm{H}$ gene) " 
Table 9: Relation between salmonella biofilm formation, Antimicrobial resistance and multidrug resistance.

\begin{tabular}{|c|c|c|}
\hline & Negative biofilm formation salmonella & Positive biofilm formation salmonella \\
\hline Biofilm formation & $14 / 36(38.88 \%)$ & $22 / 36(61.11 \%)$ \\
\hline $\begin{array}{l}\text { Antimicrobial } \\
\text { resistance }\end{array}$ & $14 / 14(100 \%)$ & $22 / 22(100 \%)$ \\
\hline Multidrug resistance & $10 / 14(71 \%)$ & $17 / 22(72 \%)$ \\
\hline $\begin{array}{l}\text { Severity in clinical } \\
\text { signs and } \\
\text { Postmortem }\end{array}$ & $\begin{array}{l}\text { 1-High degree of mortality and morbidity } \\
\text { in farm infected with salmonella. } \\
\text { 2-Signs of depression and diarrhea } \\
\text { 3-Omphilitis in young chicks } \\
\text { 4-Perhepatitis, pericarditis. } \\
\text { 5-Inflammation in brain }\end{array}$ & $\begin{array}{l}\text { 1-High degree of mortality and morbidity } \\
\text { in farm infected with salmonella } \\
\text { 2- Signs of depression and diarrhea. } \\
\text { 3- Omphilitis young chicks } \\
\text { 4- Perhepatitis, pericarditis. } \\
\text { 5- Inflammation in brain }\end{array}$ \\
\hline
\end{tabular}

Multidrug resistance: resistance for more than 3 antibiotic groups

\section{DISCUSSION}

Some salmonella strains have ability for biofilm production $61.11 \%(22 / 36)$ and the others have n't $38.88 \%$ (14/36), also antimicrobial resistance was observed in both positive and negative biofilm formation $(100 \%)$, and resulted in that there is no relation between biofilm formation and antimicrobial resistance and multidrug resistance. Also both positive and negative biofilm formation were showed same degree of mortality and morbidity, Signs of depression, diarrhea, Omphilitis in young chicks, perhepatitis, pericarditis, Oophritis, redness in brain. The obtained results were agree with (Wang et al., 2013) who reported that no significant correlation between antimicrobial resistance and biofilm production as well as agree with (Ghasemmahdi et al., 2015) who demonstrated that all Salmonella typhimurium isolates showed a high multiple antibiotic resistant with low biofilm formation capabilities which proposed low association between biofilm formation and antibiotic resistance of a major food important pathogen. As well as the results were agree with (Apellanis et al., 2017) who reported that no relationship was found between biofilm production and antimicrobial resistance in Salmonella enteritidis strains. While the present results were disagree with (Costerton et al., 1999, Hall-Stoodley et al., 2004) that demonstrated that biofilms were important factors in antimicrobial resistance, and play a key role in the pathogenesis of many bacterial infections. Bacteria with biofilms are inherently protected from their surrounding environment and often exhibit increased resistance to host defense and antimicrobial agents, making these infections difficult or impossible to eradicate. (Arciola et al., 2001, Costerton et al., 2003 and Szomolay et al., 2005) demonstrated that bacteria with biofilms may have an increased resistance to antimicrobials, ambient pressure and the host immune system, also disagree with Gong et al. (2013), reported that the proportion of biofilm-positive Salmonella pullorum isolates increased over time. The antimicrobial resistance rates of positive isolates were higher than those of negative isolates. The proportion of multidrug resistance for positive and negative biofilm formation isolates was no significant different.

In conclusion, no correlation between biofilm production and multidrug resistant in examined isolates.

\section{REFERENCE}

Apellanis, K.B.; Thales Quedi, F.T; Neves, D.S.S.; Rafaela, M.; Pippi, S.C.T.; Luiz, D.S.M.H.; César, T.E. and Pinheiro, D.N.V. (2017): Phenotypic and Molecular Characterization of Salmonella Enteritidis SE86 Isolated from Poultry and Salmonellosis Outbreaks Foodborne Pathogens and Disease, 14 (12): 742-754.

Arciola, C.R.; Baldassarri, L. and Montanaro, $\quad$ L. (2001): Presence of icaA and icaD genes and slime production in a collection of Staphylococcal strains from catheterassociated infections, Journal of Clinical Microbiology, 39: 2151-2156.

Barnhart, M.M. and Chapman, M.R. (2006): Curli biogenesis and function., Annu Rev Microbiol, 60: 131-147.

Boddicker, J.D.; Ledeboer, N.A.; Jagnow, J.; jones, B.D. and Clegg, S. (2002): Differential binding to and biofilm formation on, HEp-2 cells b salmonella enteric serovar Tphimurium is dependent upon allelic variation in the fim $\mathrm{H}$ gene of the fim gene cluster, Microbiol, 45: 1255-1265.

Costerton, J.W.; Stewart, P.S. and Greenberg, E.P. (1999): Bacterial biofilms: a common cause of persistent infections, Science, 284: 1318-1322.

Costerton, W.; Veeh, R.; Shirtliff, M.; Pasmore, M.; P ost, C. and Ehrlich, G. (2003): The application of biofilm science to the study and control of chronic bacterial infections, Journal of Clinical Investigation, 112: 1466-1477.

Chavant, P.B.; Martinie, T.; Meylheuc, T.; BellonFontaine, M. and Hebraud, M. (2002): Listeria monocytogenes LO28: surface physicochemical properties and ability to form 
biofilms at different temperatures and growth phases, Appl. Environ. Microbiol.68:728-737.

CLSI/NCClS (2009): Performance Standards for Antimicrobial Disk Susceptibility Tests; Approval Standard-Tenth Edition and Performance Standards for Antimicrobial Susceptibility Test; M02-A10 and M100-S20.

Daxin Peng (2016): Microbial Biofilms - Importance and Applications, Immunology and Microbiology, 232-249.

Donlan, R.M. and Costerton, J.W. (2002): Biofilms: survival mechanisms of clinically relevant microorganisms, Clin. Microbiol. Rev.15:167193.

Ghasemmahdi, H.; Tajik, H.; Moradi, M.; Mardani, K.; Modaresi, R.; Badali, A.; Dilmaghani, M. (2015): Antibiotic Resistance Pattern and Biofilm Formation Ability of Clinically Isolates of Salmonella enterica Serotype typhimurium, Int. J. Enteric Pathog. 3(2): e27372.

Gjermansen, M.; Ragas, P.; Sternberg, C.; Molin, S. and Tolker-Nielsen, T. (2005): Characterization of starvation-induced dispersion in Pseudomonasputida biofilms, Environ Microbiol, (7): 894-906.

Gong, J; Xu, M.; Zhu, C.; Miao, J.; Liu, X. and Xu, B. (2013): Antimicrobial resistance, presence of integrons and biofilm formation of Salmonella Pullorum isolates from eastern China (1962-2010) Avain pathology, Pages 290-294.

Hall-Stoodley, L.; Costerton, J.W. and Stoodley, P. (2004): Bacterial biofilms: from the natural environment to infectious diseases. Nature Reviews Microbiology, 2: 95-108.

Hojati, Z.; Zamanzad, B.; Hashemzadeh, M.; Molaie, R. and Abolfazl Gholipour, A. (2015):
Detection of Fim H Gene in Uropathogenic Escherichia coli Strains isolated From Patients With Urinary Tract Infection, J. Microbiol.; 8(2): e17520.

ISO (6579:2002): Microbiology of food and animal feeding stuffs - Horizontal method for the detection of Salmonella. ISO 6579: 2002(E) International Standards Organization, Geneva

Kadlec, K.; Michael, G.B.; Fessler, A.T. and Schwarz, S. (2012): Molecular mechanisms of antibiotic resistances. Antibiotics in Food Chain., Proceedings of the Max Rubner Conference; Karlsruhe. Netherlands. p.16.

Ledeboer, N.A. and Jones, B.D. (2005): Exopolysaccharide sugars contribute to biofilm formation by Salmonella enteric serovartyphimurium on HEp-2 cells and chicken intestinal epithelium. J. Bacteriol, 187: 3214-3226.

Majowicz, S.E.; Musto, J.; Scallan, E.; Angulo, F.J.; Kirk, M. and O'Brien, S.J. (2010): The global burden of nontyphoidal Salmonella gastroenteritis, Clin. Infect. Dis. 50: 882-889.

Popoff, M.Y. (2001): Antigenic formulas of the Salmonella Serovars, WHO Collaborating Center for reference and research on Salmonella, Paris. (8th edition).

Szomolay, B.; Klapper, I.; Dockery, J. and Stewart, P.S. (2005): Adaptive responses to antimicrobial agents in biofilms, Environmental Microbiology, 7: 1186-1191.

Wang, H.; Ye, K.; Wei, X.; Cao, J; Xu, X. and Zho, G. (2013): Occurrence, antimicrobial resistance and biofilm formation of Salmonella isolates from a chicken slaughter plant in China, Food Control, 33: (2), 378-384.

\title{
الكثف عن تكوين البيوفيلم ومقاومة المضادات الحيوية للسالمونيلا في دجاج التسمين \\ هند كرم عبل السلام سرور ، فاطمة عامر عبل الرحيم
}

E-mail:drhendsorour@gmail.com Assiut University web-site: www.aun.edu.eg

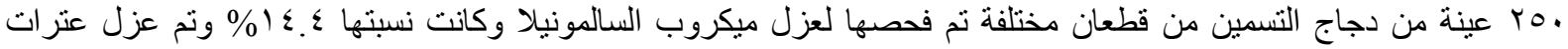

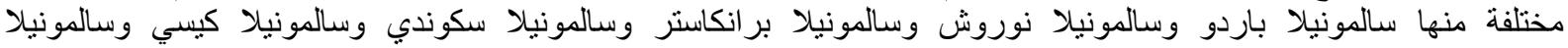

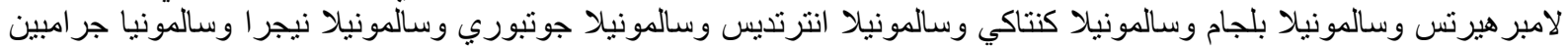

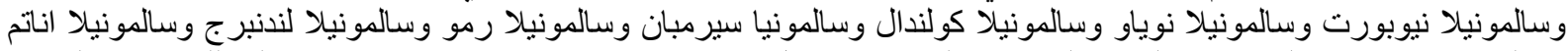

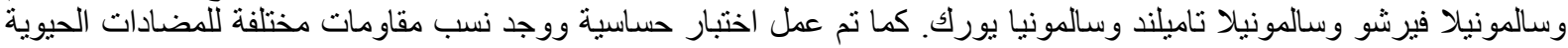

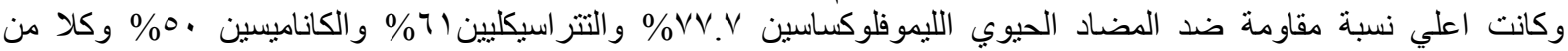

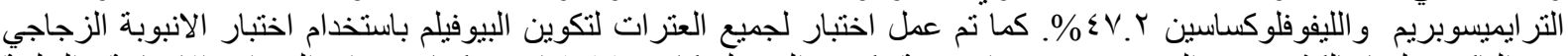

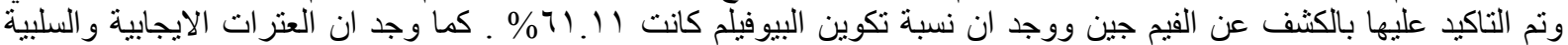

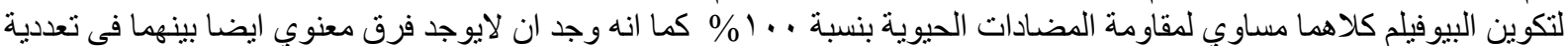

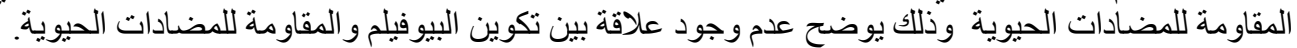

